\title{
Quantification of the type 2 diabetes risk in women with gestational diabetes: a systematic review and meta-analysis of 95,750 women
}

\author{
Girish Rayanagoudar ${ }^{1}$ • Amal A. Hashi ${ }^{1}$. Javier Zamora ${ }^{1,2,3}$ - Khalid S. Khan ${ }^{1,4}$. \\ Graham A. Hitman ${ }^{1}$ • Shakila Thangaratinam ${ }^{1,4}$
}

Received: 21 November 2015 / Accepted: 25 February 2016/Published online: 13 April 2016

(C) The Author(s) 2016. This article is published with open access at Springerlink.com

\begin{abstract}
Aims/hypothesis Women with gestational diabetes mellitus (GDM) are at risk of developing type 2 diabetes, but individualised risk estimates are unknown. We conducted a meta-analysis to quantify the risk of progression to type 2 diabetes for women with GDM.

Methods We systematically searched the major electronic databases with no language restrictions. Two reviewers independently extracted $2 \times 2$ tables for dichotomous data and the means plus SEs for continuous data. Risk ratios were calculated and pooled using a random effects model.

Results There were 39 relevant studies (including 95,750 women) BMI (RR 1.95 [95\% CI 1.60, 2.31]), family history of diabetes (RR 1.70 [95\% CI 1.47, 1.97]), non-white ethnicity (RR 1.49 [95\% CI 1.14, 1.94]) and advanced maternal age (RR $1.20[95 \%$ CI 1.09, 1.34]) were associated with future risk of type 2 diabetes. There was an increase in risk with early diagnosis of GDM (RR 2.13 [95\% CI 1.52, 3.56]), raised
\end{abstract}

Electronic supplementary material The online version of this article (doi:10.1007/s00125-016-3927-2) contains peer-reviewed but unedited supplementary material, which is available to authorised users.

Shakila Thangaratinam

s.thangaratinam@qmul.ac.uk

1 Women's Health Research Unit, Blizard Institute, Barts and The London School of Medicine and Dentistry, Queen Mary University of London, 4 Newark Street, London E1 2AB, UK

2 Clinical Biostatistics Unit, Hospital Ramon y Cajal (IRYCIS), Madrid, Spain

3 CIBER Epidemiologia y Salud Publica (CIBERESP), Madrid, Spain

4 Multidisciplinary Evidence Synthesis Hub (mEsh), Blizard Institute, Barts and The London School of Medicine and Dentistry, Queen Mary University of London, London, UK fasting glucose (RR 3.57 [95\% CI 2.98, 4.04]), increased $\mathrm{HbA}_{1 \mathrm{c}}$ (RR 2.56 [95\% CI 2.00, 3.17]) and use of insulin (RR 3.66 [95\% CI 2.78, 4.82]). Multiparity (RR $1.23[95 \%$ CI 1.01, 1.50]), hypertensive disorders in pregnancy (RR 1.38 [95\% CI 1.32, 1.45]) and preterm delivery (RR 1.81 [95\% CI $1.35,2.43])$ were associated with future diabetes. Gestational weight gain, macrosomia in the offspring or breastfeeding did not increase the risk.

Conclusions/interpretation Personalised risk of progression to type 2 diabetes should be communicated to mothers with GDM.

Systematic review registration: www.crd.york.ac.uk/ PROSPERO CRD42014013597

Keywords Gestational diabetes · Meta-analysis ·

Postpartum $\cdot$ Predictors $\cdot$ Pregnancy $\cdot$ Risk factors $\cdot$ Systematic review $\cdot$ Type 2 diabetes

$\begin{array}{ll}\text { Abbreviations } \\ \text { GDM } & \text { Gestational diabetes mellitus } \\ \text { IPD } & \text { Individual participant data } \\ \text { PRISMA } & \text { Preferred Reporting Items for Systematic } \\ & \text { Reviews and Meta-Analyses }\end{array}$

\section{Introduction}

Gestational diabetes mellitus (GDM), defined as glucose intolerance that is first diagnosed in pregnancy, is on the increase worldwide [1]. Up to half of all women with this condition progress to develop type 2 diabetes in later life, with the highest occurrence rate in the first 5 years after pregnancy [2]. Increasing numbers of women are presenting with previously undiagnosed diabetes and related complications, leading to substantial healthcare costs [3]. 
Current guidelines recommend following up women with GDM to identify type 2 diabetes at an early stage [4]. Ensuring compliance with this strategy is a significant global problem [5-7]. Despite evidence that the future risk of type 2 diabetes can be reduced by diet and lifestyle interventions and treatment with drugs such as metformin, [8,9], less than a fifth of mothers with GDM undergo postpartum glucose screening [10]. Personalised risk communication with quantitative estimates can increase the number of individuals that make informed choices in screening programmes [11]. However, few prediction models for type 2 diabetes include GDM, and none of them account for pregnancy-specific characteristics [12].

Gestation-specific factors such as glycaemic status in pregnancy, gestational weight gain and obstetric complications are known to influence the future risk of diabetes [13-17]. Healthcare professionals involved in the management of women with GDM do not provide individual risk estimates because of the lack of data; this greatly hinders counselling. Existing reviews have not quantified the risk of progression to type 2 diabetes $[16,18]$, and primary studies provide imprecise estimates because of their relatively small sample size [19-21]. We therefore undertook a systematic review to assess the strength of association of various maternal and pregnancyrelated factors with GDM with the future risk of type 2 diabetes.

\section{Methods}

We conducted a systematic review following a prospective protocol in line with current recommendations, and complied with the Preferred Reporting Items for Systematic Reviews and Meta-Analyses (PRISMA) guidelines for reporting (see electronic supplementary material [ESM] PRISMA checklist) [22]. Ethical approval was not required.

Data sources and searches We searched the MEDLINE and EMBASE databases (from inception until July 2015) for studies that assessed the risk factors in women with GDM and progression to type 2 diabetes. We used keywords, Medical Subject Headings and word variants for GDM such as 'diabetes, gestational', 'GDM', and 'pregnancy induced diabetes', and combined these with terms for type 2 diabetes such as 'diabetes mellitus, type 2', 'NIDDM' (i.e. non-insulindependent diabetes mellitus), 'adult-onset diabetes mellitus' and 'ketosis-resistant diabetes' (See ESM Search strategy). We searched the reference lists of identified papers for other relevant studies. Authors of eligible studies were contacted for further details if necessary. We did not apply any language restrictions.

Study selection Two independent reviewers (GR and AAH) selected the studies. First, we reviewed the abstracts for potentially relevant studies. Second, we obtained full copies of all possibly eligible papers for detailed evaluation. We included studies on women with GDM that assessed at least one of the following factors: maternal characteristics such as age, BMI, ethnicity, parity and family history of type 2 diabetes; factors specific to pregnancy such as gestational age at GDM diagnosis, antenatal OGTT, $\mathrm{HbA}_{1 \mathrm{c}}$, insulin use in pregnancy, gestational weight gain, pregnancy-induced hypertension and preterm delivery; and postpartum factors such as the baby's birthweight and breastfeeding. We excluded studies with no relevant data, an inappropriate outcome, no original data (e.g. meeting abstract, editorial, commentary or letter) or duplicate data. We accepted the authors' definitions of GDM and type 2 diabetes. Any discrepancies in selection were resolved by discussion with a third reviewer (ST).

Data extraction and quality assessment Two reviewers (GR and $\mathrm{AAH}$ ) independently undertook study quality assessment and data extraction using predesigned forms. We used the Newcastle-Ottawa scale [23], which evaluates the representativeness and selection of studies, comparability among cohorts, ascertains the exposure and outcome, and evaluates the length and adequacy of follow-up. The risk of bias was considered to be low if a study obtained four stars for selection, two for comparability and three for follow-up; and medium if a study scored two or three stars for selection, one for comparability and two for follow-up. Studies scoring one or zero stars for selection and follow-up, and with no star for comparability, were deemed to have a high risk of bias [24]. Data were extracted as $2 \times 2$ tables for dichotomous outcomes, and as means and SEs for continuous outcomes.

Data synthesis and analysis For various risk factors, we calculated the RRs for dichotomous variables, and plotted point estimates and $95 \%$ CIs for progression to type 2 diabetes for women with GDM associated with various risk factors. For continuous variables, we plotted pooled mean differences with 95\% CIs to assess differences between women with and without type 2 diabetes. We assessed the heterogeneity of association graphically with forest plots and statistically with $\chi^{2}$ tests and the $I^{2}$ statistic. We pooled results using random effects models. To facilitate comparison between the strength of association of various risk factors with type 2 diabetes, we transformed the pooled standardised mean differences of continuous outcomes into RRs, assuming a $20 \%$ baseline risk of type 2 diabetes [25].

We specified the following subgroup analyses a priori: length of follow-up ( $\leq 1$ year vs $>1$ year), ethnicity (white vs non-white), use of strict criteria to exclude possible preexisting type 2 diabetes (such as GDM before 20 weeks of pregnancy or type 2 diabetes diagnosis in the first year after delivery; yes vs no). Additionally, we investigated differences between subgroups based on the levels of fasting glucose criteria for diagnosing GDM $(<5.8 \mathrm{mmol} / \mathrm{l}$ vs $\geq 5.8 \mathrm{mmol} / \mathrm{l})$. 
All analyses were performed using the Review Manager (RevMan Computer program, Version 5.2; The Nordic Cochrane Centre, Copenhagen, Denmark) [26].

\section{Results}

Study identification From 5,966 citations, we selected 178 studies for further evaluation (Fig. 1). After a detailed assessment, we included 39 studies (95,750 women) [13-15, 17, 19-21, 27-58]. We excluded 139 studies for the following reasons: inclusion criteria were not met $(n=65)$; presented as posters, conference abstracts or letters $(n=50)$; inappropriate data format $(n=15)$; only abstracts were available $(n=4)$; duplicate data $(n=4)$; and could not be translated from the language of publication $(n=1)$.

Characteristics and quality of the included studies In all, there were 22 prospective cohort studies $(56 \%)$ [13, 14, 17, 19-21, 29, 32, 35-37, 39-41, 43, 44, 46-48, 50, 52, 55] and 17 retrospective cohort studies (44\%) [15, 27, 28, 30, 31, 33, $34,38,42,45,49,51,53,54,56-58]$. The studies evaluated the association of maternal characteristics, pregnancy-specific factors and postpartum characteristics with progression to type 2 diabetes in women with GDM. The studies varied in their

Fig. 1 Flow chart for study selection for the systematic review of the predictors of progression to type 2 diabetes in women with GDM diagnostic criteria for both GDM and type 2 diabetes. Maternal characteristics including BMI, ethnicity and family history of diabetes were evaluated, as were risk factors specific to pregnancy such as maternal age at diagnosis of GDM, parity, gestational age at diagnosis, weight gain in pregnancy, hypertensive diseases in pregnancy and preterm birth, and levels of fasting, post-load blood glucose levels during OGTT, $\mathrm{HbA}_{1 \mathrm{c}}$ in pregnancy and use of insulin for managing GDM.

The duration of follow-up varied from 6 weeks to 20 years after birth. A total of 29 studies (74\%) evaluated the long-term risk of type 2 diabetes ( $>1$ year after delivery) and $10(26 \%)$ evaluated the risk of developing type 2 diabetes in the first year after childbirth. Detailed characteristics of the included studies are provided in ESM Table 1. In all, six studies (15\%) had a low risk of selection bias, $32(82 \%)$ had a medium risk and one (3\%) had a high risk. Eight studies (21\%) had a low risk of bias for comparability of cohorts, and $31(79 \%)$ had a medium risk of bias. For outcome assessment, 11 studies $(28 \%)$ had a low risk of bias, $22(56 \%)$ had a medium risk and $6(15 \%)$ had a high risk (see Fig. 2 and ESM Table 2).

Maternal characteristics and progression to type $\mathbf{2}$ diabetes A high BMI doubled the risk of future type 2 diabetes (RR $1.95[95 \%$ CI $1.60,2.31] ; I^{2}=65 \%$ ), and the risk was

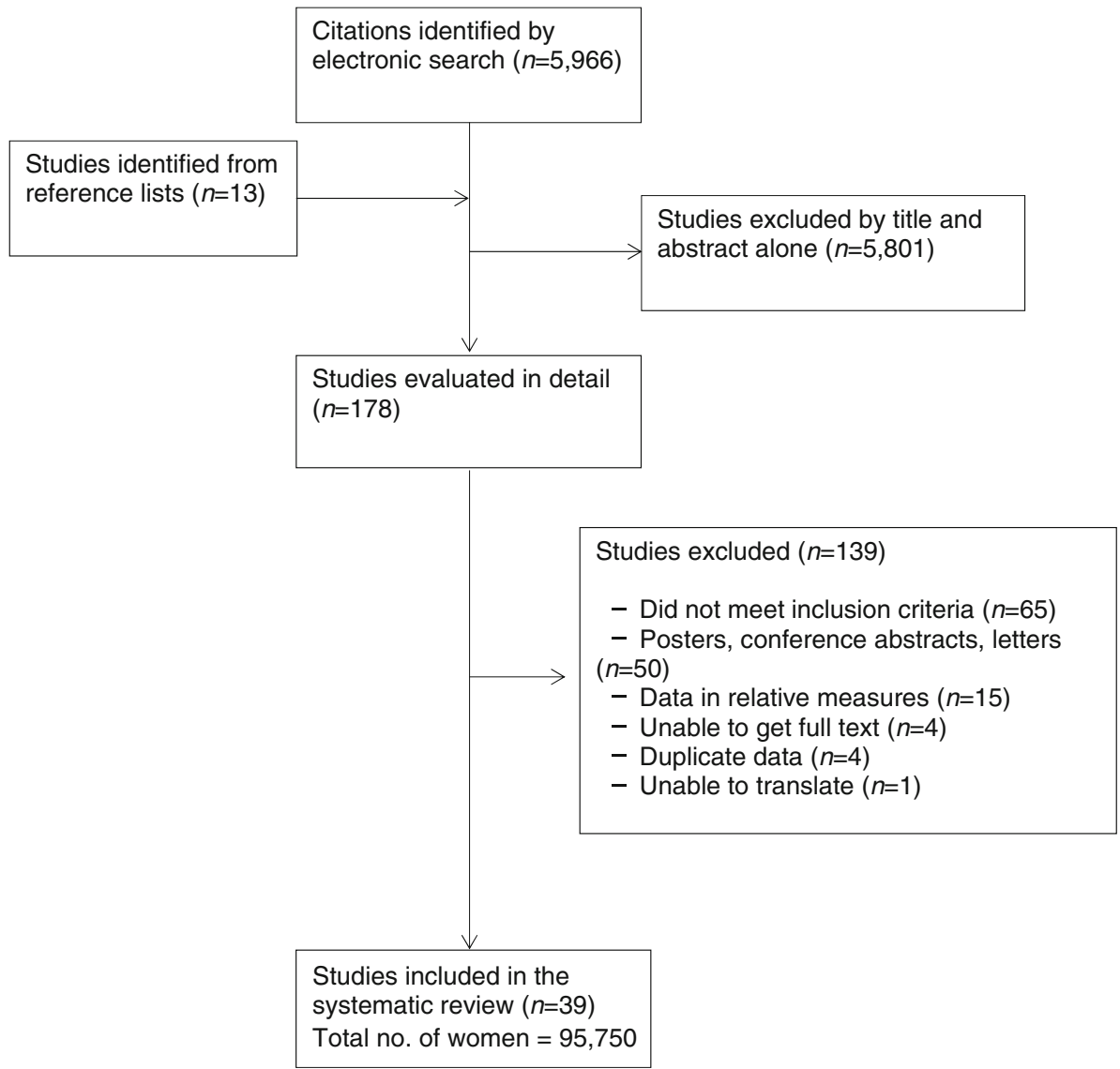




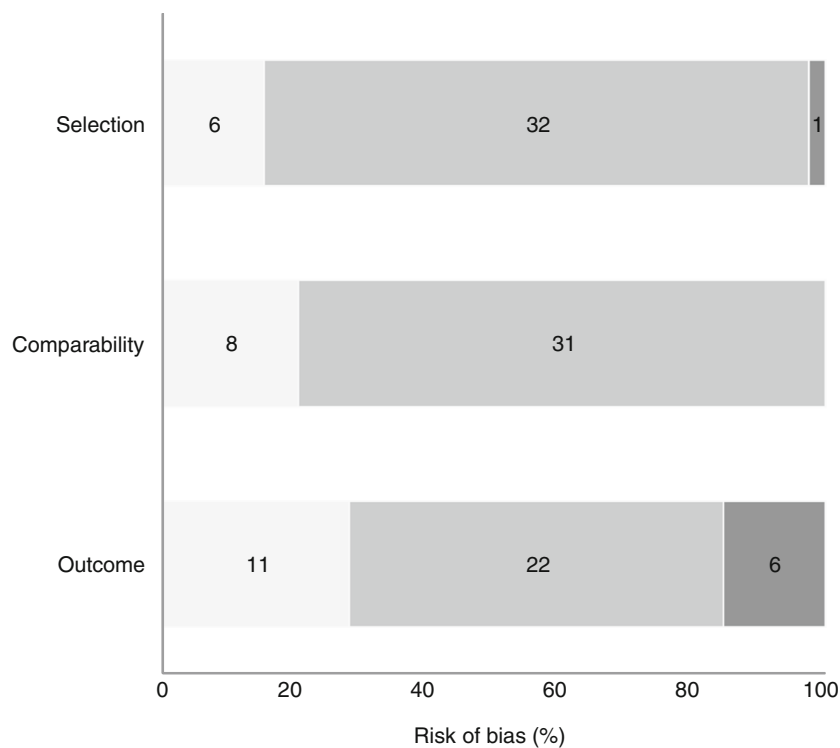

Fig. 2 Risk of bias assessment on the Newcastle-Ottawa Scale for studies included in the systematic review of type 2 diabetes prediction in women with GDM. Light grey bars, low risk; mid grey bars, medium risk; dark grey bars, high risk. The numbers of studies are shown

increased in obese and overweight women for BMI thresholds of $25 \mathrm{~kg} / \mathrm{m}^{2}$ (RR 3.18 [95\% CI 1.96, 5.16]; $I^{2}=77 \%$ ), $27 \mathrm{~kg} / \mathrm{m}^{2}$ (RR 2.52 [95\% CI 1.69, 3.74]; $I^{2}=23 \%$ ) and $30 \mathrm{~kg} / \mathrm{m}^{2}$ (RR $2.85[95 \%$ CI $\left.2.21,3.69] ; I^{2}=45 \%\right)$. A family history of diabetes (RR 1.70 [95\% CI 1.47, 1.97]; $I^{2}=13 \%$ ), non-white ethnicity (RR 1.49 [95\% CI 1.14, 1.94]; $I^{2}=88 \%$ ) and older age (RR 1.20 [95\% CI 1.09, 1.34]; $I^{2}=0 \%$ ) were found to be significant risk factors for progression to diabetes after GDM. No increased risk was observed for individual age cut-offs of 30 and 35 years (Fig. 3).

Pregnancy-specific factors and risk of future diabetes Increased levels of fasting (RR 3.57; 95\% CI 2.98, 4.04; $\left.I^{2}=90 \%\right), 1 \mathrm{~h}\left(\mathrm{RR} 3.05 ; 95 \%\right.$ CI $\left.2.40,3.63 ; I^{2}=64 \%\right), 2 \mathrm{~h}$ (RR 3.46; 95\% CI 2.60, 4.10; $I^{2}=80 \%$ ) and $3 \mathrm{~h}$ (RR 3.2; $95 \%$ CI $2.54,3.75 ; I^{2}=65 \%$ ) blood glucose levels after OGTT and high $\mathrm{HbA}_{1 \mathrm{c}}$ were associated with an increased risk of future diabetes (RR 2.56; 95\% CI 2.00, 3.17; $I^{2}=54 \%$ ). Women who required insulin to manage GDM were more likely to develop type 2 diabetes (RR 3.66 [95\% CI 2.78, 4.82]; $I^{2}=71 \%$ ) compared with those managed without insulin (Fig. 3).

Multiparity was a significant risk factor compared with nulliparity (RR 1.23 [95\% CI 1.01, 1.50]; $I^{2}=0 \%$ ). Women with pregnancy complications such as hypertensive disease (RR 1.38 [95\% CI 1.32, 1.45]; $I^{2}=0 \%$ ) and preterm delivery ( $<37$ weeks) (RR $1.81[95 \%$ CI $1.35,2.43] ; I^{2}=0 \%$ ) were more likely to develop type 2 diabetes in the future. There were no differences in gestational weight gain (mean difference $-0.83 \mathrm{~kg}[95 \% \mathrm{CI}-2.18,0.51] ; I^{2}=65 \%$ ) between the two groups (ESM Fig. 1).
Delivery and postpartum factors The risk of developing type 2 diabetes was not associated with birthweight (RR 1.19 [95\% CI $0.86,1.58] ; I^{2}=60 \%$ ), fetal macrosomia (RR $0.91[95 \%$ CI $0.44,1.86]$ ) or breastfeeding (RR 0.77 [95\% CI $\left.0.48,1.22] ; I^{2}=9 \%\right)$.

Subgroup analysis There were significant between-group differences based on follow-up time ( $<1$ year vs $>1$ year) for risk factors such as fasting glucose $(p=0.04)$, BMI $(p=0.03)$ and insulin use $(p=0.006)$ and type 2 diabetes. We did not observe any between-group differences based on ethnicity and GDM diagnostic criteria (fasting glucose level of $<5.8 \mathrm{mmol} / 1$ or $\geq 5.8 \mathrm{mmol} / \mathrm{l}$ ) for predictors such as maternal age, BMI, family history of diabetes, need for insulin in pregnancy and the risk of type 2 diabetes. There was a significant difference between subgroups based on strict criteria for excluding possible pre-existing type 2 diabetes for fasting glucose as a predictor of type 2 diabetes $(p=0.02)$, but no differences were observed for associations with other predictors (Table 1).

\section{Discussion}

Summary of findings The future risk of diabetes appears to be mainly influenced by the gestational glycaemic status, and not by the mother's gestational weight gain or baby's birthweight. We found that both hypertensive disorders in pregnancy and preterm delivery in GDM pregnancies were associated with future onset of type 2 diabetes, which was previously unknown. Factors specific to pregnancy such as gestational age at onset of GDM and general maternal characteristics such as BMI, ethnicity and family history were also associated with future onset of type 2 diabetes. This systematic review has thus collated the information necessary for postnatal counselling of women with GDM.

Strengths and limitations To our knowledge, this is the first review to quantify the links between clinical characteristics (especially those relevant to pregnancy) and the future onset of diabetes in women with GDM. This systematic review used a prospective protocol and a comprehensive search without any language restrictions. Our meta-analysis included a large number of studies, and we were able to study most of the potentially relevant risk factors. Previous reviews were limited in their findings by the small number of studies included and the absence of summary estimates $[2,16,18,59]$. We have provided clinically relevant estimates as RRs for use in counselling of women.

The studies included had varying definitions of population, risk factors and methods of ascertaining the outcome, which included different lengths of follow-up. Subgroup analysis was planned a priori to identify any sources of heterogeneity. It is possible that some studies could have misclassified preexisting type 2 diabetes as GDM, especially when the 


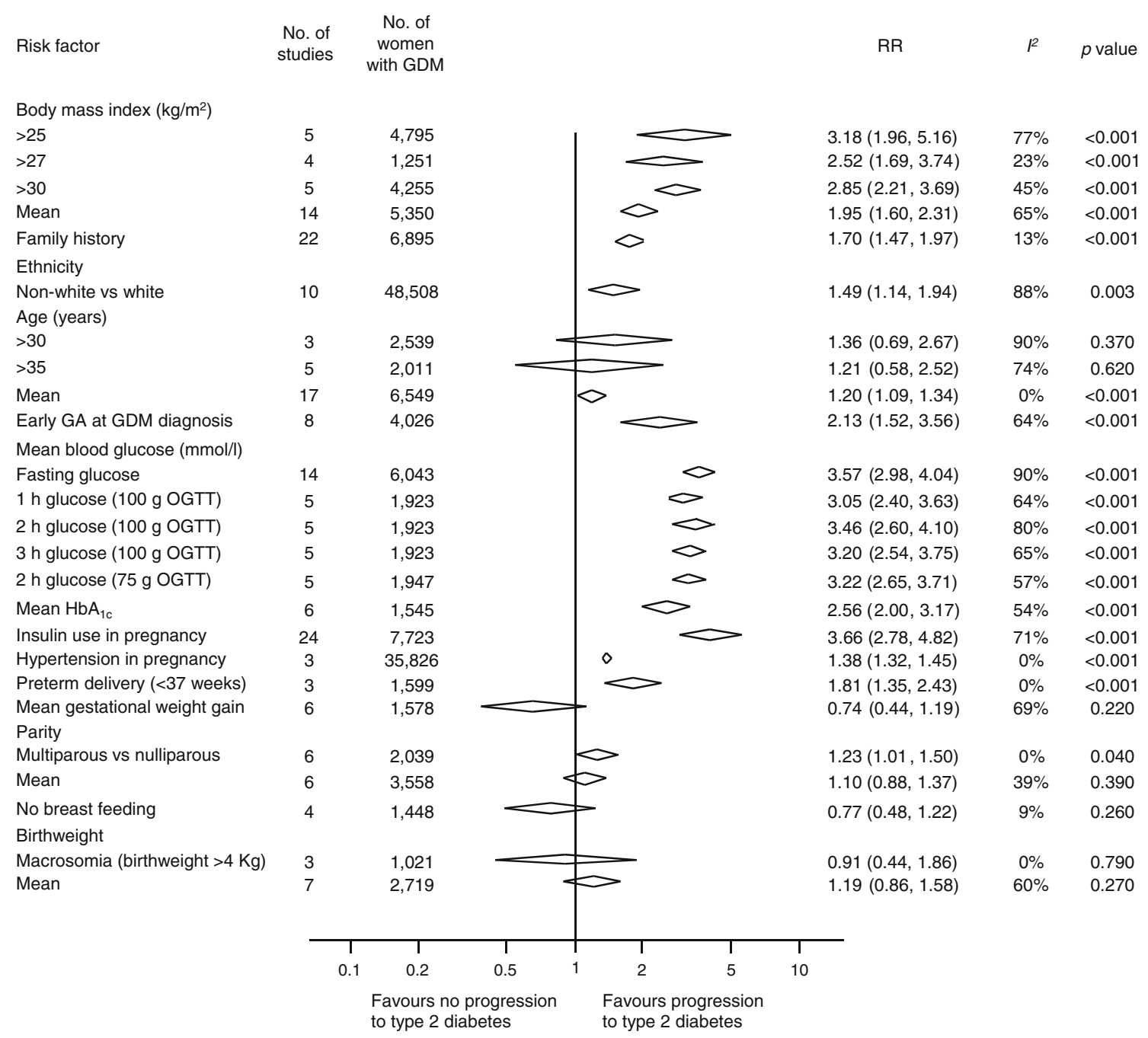

Fig. 3 Summary estimates for the association of maternal risk factors with progression to type 2 diabetes in women with GDM. 'Exclusively' and 'mostly' breastfed were combined into a single breastfeeding category. 'Mixed or inconsistent' breastfeeding and 'exclusively or mostly formula fed' were combined into a single 'no breastfeeding' category [17].

diagnosis was made as early as 6 weeks after delivery. However, we did not observe significant between-group differences based on strict criteria for excluding pre-existing type 2 diabetes (studies that excluded women with GDM diagnosed within 20 weeks' gestation or diabetes in the first year after delivery vs studies that did not). Some studies included only diet-controlled GDM, and this may have underestimated the risk of progression to type 2 diabetes.

The studies varied in the criteria used for diagnosing GDM and in the thresholds for commencing insulin in pregnancy; a third used Carpenter and Coustan criteria for diagnosing GDM. Our subgroup analysis was based on a fasting glucose cut-off value of $5.8 \mathrm{mmol} / \mathrm{l}: 69 \%$ of studies under this cut-off used Carpenter and Coustan criteria, and showed no significant differences in estimates for progression based on the criteria used for GDM diagnosis. Despite these variations,
Similarly, data reported for age $<34$ years in one study [37] was categorised as age $<35$ years, and data reported as BMI of $<28 \mathrm{~kg} / \mathrm{m}^{2}$ for one study [50] was classified as a BMI of $<27 \mathrm{~kg} / \mathrm{m}^{2}$ because all other studies used this cut-off value. GA, gestational age

we consistently observed an increased risk of future diabetes in women with gestational hyperglycaemia.

We evaluated associations of individual predictors with outcomes, but could not adjust for confounding variables such as BMI because of the lack of access to individual data. The lack of detail in reported data made it difficult to evaluate the simultaneous influence of multiple factors on outcome. However, we could provide robust, precise estimates for individual risk factors that are relevant for providing postnatal information to women with GDM.

Comparison with existing evidence In the non-pregnant state, BMI, family history of diabetes and ethnicity are associated with the risk of type 2 diabetes [18, 60-63]. We observed the same in mothers with GDM, and there was a greater risk of progression in non-white than in white women. Individual 
Table 1 Subgroup analysis for progression to type 2 diabetes after GDM by follow-up time, ethnicity, timing of diagnosis and fasting glucose criteria for GDM diagnosis

\begin{tabular}{|c|c|c|c|c|c|c|c|c|c|c|c|c|c|}
\hline \multirow[t]{2}{*}{$\begin{array}{l}\text { Risk } \\
\text { factor }\end{array}$} & \multicolumn{3}{|l|}{ Follow-up } & \multicolumn{4}{|l|}{ Ethnicity } & \multicolumn{3}{|c|}{$\begin{array}{l}\text { Strict criteria used to exclude } \\
\text { pre-existing type } 2 \text { diabetes? }^{\text {a }}\end{array}$} & \multicolumn{3}{|c|}{$\begin{array}{l}\text { Fasting glucose for } \\
\text { GDM diagnosis }\end{array}$} \\
\hline & $<1$ year & $\geq 1$ year & $\begin{array}{l}p \\
\text { value }\end{array}$ & White & $\begin{array}{l}\text { Non- } \\
\text { white }\end{array}$ & Mixed & $\begin{array}{l}p \\
\text { value }\end{array}$ & Yes & No & $\begin{array}{l}p \\
\text { value }\end{array}$ & $\begin{array}{l}<5.8 \\
\mathrm{mmol} / 1\end{array}$ & $\begin{array}{l}\geq 5.8 \\
\mathrm{mmol} / 1\end{array}$ & $\begin{array}{l}p \\
\text { value }\end{array}$ \\
\hline Age & $\begin{array}{l}1.27[1.11 \\
1.45]\end{array}$ & $\begin{array}{c}1.14[0.97, \\
1.32]\end{array}$ & 0.29 & $\begin{array}{l}1.29[0.99 \\
1.64]\end{array}$ & $\begin{array}{l}1.19[0.97, \\
1.41]\end{array}$ & $\begin{array}{l}1.20[1.03, \\
1.39]\end{array}$ & 0.85 & $\begin{array}{l}1.11[0.81 \\
1.45]\end{array}$ & $\begin{array}{l}1.15[0.96 \\
1.38]\end{array}$ & 0.79 & $\begin{array}{l}1.47[0.92 \\
2.17]\end{array}$ & $\begin{array}{l}1.15[1.01 \\
1.29]\end{array}$ & 0.32 \\
\hline $\begin{array}{l}\text { Fasting } \\
\text { glucose }\end{array}$ & $\begin{array}{l}4.13[3.32 \\
\quad 4.59]\end{array}$ & $\begin{array}{l}3.13[2.54 \\
3.65]\end{array}$ & 0.04 & $\begin{array}{l}4.13[1.43 \\
\quad 4.91]\end{array}$ & $\begin{array}{l}3.34[2.78 \\
3.83]\end{array}$ & $\begin{array}{l}3.38[2.54 \\
4.03]\end{array}$ & 0.80 & $\begin{array}{l}2.40[1.89 \\
2.94]\end{array}$ & $\begin{array}{l}3.48[2.76 \\
\quad 4.04]\end{array}$ & 0.02 & - & - & - \\
\hline BMI & $\begin{array}{c}1.56[1.15 \\
2.04]\end{array}$ & $\begin{array}{c}2.20[1.89 \\
2.51]\end{array}$ & 0.03 & $\begin{array}{c}2.11[1.29 \\
3.05]\end{array}$ & $\begin{array}{c}2.06[1.66 \\
2.51]\end{array}$ & $\begin{array}{c}1.74[1.24 \\
2.33]\end{array}$ & 0.63 & $\begin{array}{c}1.95[1.47 \\
2.47]\end{array}$ & $\begin{array}{c}2.38[2.04 \\
2.74]\end{array}$ & 0.18 & $\begin{array}{c}1.98[1.39 \\
2.60]\end{array}$ & $\begin{array}{c}1.87[1.41 \\
2.40]\end{array}$ & 0.81 \\
\hline $\begin{array}{l}\text { Family } \\
\text { history }\end{array}$ & $\begin{array}{c}1.63[1.19 \\
2.24]\end{array}$ & $\begin{array}{c}1.74[1.46 \\
2.07]\end{array}$ & 0.72 & $\begin{array}{c}1.49[1.20 \\
1.85]\end{array}$ & $\begin{array}{c}1.78[1.35 \\
2.35]\end{array}$ & $\begin{array}{c}1.95[1.45, \\
2.62]\end{array}$ & 0.31 & $\begin{array}{c}1.80[1.27 \\
2.54]\end{array}$ & $\begin{array}{c}1.73[1.39 \\
2.15]\end{array}$ & 0.86 & $\begin{array}{c}1.83[1.25 \\
2.66]\end{array}$ & $\begin{array}{c}1.72[1.42, \\
2.09]\end{array}$ & 0.78 \\
\hline Insulin & $\begin{array}{c}7.19[4.14 \\
12.48]\end{array}$ & $\begin{array}{c}3.06[2.39, \\
3.92]\end{array}$ & 0.006 & $\begin{array}{c}3.49[2.25 \\
5.40]\end{array}$ & $\begin{array}{c}4.09[2.27, \\
7.34]\end{array}$ & $\begin{array}{c}3.37[2.43, \\
4.67]\end{array}$ & 0.85 & $\begin{array}{c}2.62[1.70 \\
4.02]\end{array}$ & $\begin{array}{c}3.43[2.62 \\
4.49]\end{array}$ & 0.29 & $\begin{array}{c}4.37[2.46 \\
\quad 7.76]\end{array}$ & $\begin{array}{c}4.46[3.63, \\
5.49]\end{array}$ & 0.95 \\
\hline
\end{tabular}

Data are RR (95\% CIs)

${ }^{\mathrm{a}}$ Diagnosis of GDM before 20 weeks of gestation or type 2 diabetes less than 1 year after delivery

studies have shown that particular ethnic groups such as blacks and South Asians are at an increased risk [15, 34]. Owing to the various classifications of ethnicity used in primary studies, we were unable to provide progression rates for specific ethnic subgroups, which may have contributed to the heterogeneity.

We did not identify an increased risk for women with high weight gain during pregnancy. However, it is likely that women with severe GDM undergo intensive monitoring and receive significant input regarding their diet and lifestyles, thereby restricting their weight gain in pregnancy [50].

Although previous studies have shown an improvement in glucose homeostasis with breastfeeding [17, 64], we found no significant association between the absence of breast-feeding and progression to diabetes. This could be attributed to the imprecise estimates obtained owing to the small number of studies and individuals. Previous studies have shown improved glucose values on OGTTs, especially in the short term [64]. Our review measured the impact on type 2 diabetes and not on lesser degrees of glucose intolerance. Furthermore, the beneficial effect on glucose levels may not have been sustained in the long term. The rigour with which breastfeeding information was collected also varied by study; most data were self-reported by the women.

In current practice, the criteria used for diagnosing GDM are not applicable in many centres. However, given the long-term outcomes evaluated in our review, we were unable to assess for heterogeneity in estimates based on the WHO 2013 criteria for diagnosing GDM [65], which are recommended in many existing national and international guidelines.

Implications for clinical practice Pregnancy is an important point in the life of a woman when she has regular contact with the healthcare system, thus providing opportunities to influence the future health of both mother and child. One of the major factors responsible for poor postnatal screening for diabetes and subsequent follow-up has been the lack of clear communication between secondary and primary care providers $[10,66]$. Pregnancy-specific findings such as glycaemic control, despite being associated with a fourfold increase in the risk of future diabetes, are not taken into count during counselling.

Postnatal advice to women with GDM should incorporate information on their individual risk factors. Communication between hospitals and general practitioners on the mother's risk of future diabetes could be improved by providing discharge summaries with pregnancy-specific details such as OGTT results, gestational age at GDM diagnosis, use of insulin, and complications such as pre-eclampsia and preterm birth. An efficient system of data linkage and communication between secondary and primary care providers will enable general practitioners to incorporate this information into their management of mothers; in particular, the management of women at a highrisk of developing type 2 diabetes could involve measures such as reminder systems [67]. Women are more likely to comply with diet and lifestyle changes if they know their individual risks of future diabetes [68].

Research recommendations Current prediction models for type 2 diabetes should be updated and validated by including the factors identified in this review. There is a need for welldesigned, prospective long-term studies to explore the association between breastfeeding and future type 2 diabetes [64]. The meta-analysis of individual participant data (IPD) could overcome many of the limitations of our meta-analysis [69]. A large-scale IPD meta-analysis could enable us to predefine the desired clinically relevant endpoints (e.g. timing of diabetes onset) and cut-off values for clinical variables, standardise the definitions of predictors and outcomes, take into account the performance of many candidate prognostic variables, directly handle missing data on both predictors and outcomes, and account for heterogeneity in baseline risks. 
Conclusion Postnatal counselling of women with GDM should be individualised for the risk of future diabetes.

Funding This independent research was funded by European Union's Seventh Framework Programme under EC-GA No. 278917 (GIFTS 'Genomic and lifestyle predictors of fetal outcome relevant to diabetes and obesity and their relevance to prevention strategies in South Asian peoples'). The funding body played no role in designing or conducting the study or preparing the report.

Duality of interest statement All authors completed the International Committee of Medical Journal Editors (ICMJE) uniform disclosure form (www.icmje.org/coi disclosure.pdf) and declare that they have received no support from any organisation for the submitted work, have had no financial relationship over the last 3 years with any organisation that might have an interest in the submitted work, and have no other relationships or activities that could influence the submitted work.

Contribution statement GAH and KSK contributed to the study design; GR and ST designed the protocol; AAH and GR selected eligible studies; GR developed the data extraction form; AAH and GR extracted the data; ST resolved discrepancies between reviewers on text selection and data extraction; GR, JZ and ST wrote the statistical analysis plan; GR cleaned and analysed the data; GR drafted the paper, AAH, GAH, JZ, KSK and ST revised the manuscript; and ST oversaw the conduct of study at all stages. All authors read and approved the final draft before submission. GR is the guarantor of this work.

Open Access This article is distributed under the terms of the Creative Commons Attribution 4.0 International License (http:// creativecommons.org/licenses/by/4.0/), which permits unrestricted use, distribution, and reproduction in any medium, provided you give appropriate credit to the original author(s) and the source, provide a link to the Creative Commons license, and indicate if changes were made.

\section{References}

1. Galtier F (2010) Definition, epidemiology, risk factors. Diabetes Metab 36:628-651

2. Bellamy L, Casas JP, Hingorani AD, Williams D (2009) Type 2 diabetes mellitus after gestational diabetes: a systematic review and meta-analysis. Lancet 373:1773-1779

3. Hex N, Bartlett C, Wright D, Taylor M, Varley D (2012) Estimating the current and future costs of Type 1 and Type 2 diabetes in the UK, including direct health costs and indirect societal and productivity costs. Diabet Med 29:855-862

4. National Institute for Health and Care Excellence (2008) Diabetes in pregnancy: management of diabetes and its complications from preconception to the postnatal period. Clinical Guideline 63 . National Institute for Health and Care Excellence, London

5. Almario CV, Ecker T, Moroz LA, Bucovetsky L, Berghella V, Baxter JK (2008) Obstetricians seldom provide postpartum diabetes screening for women with gestational diabetes. Am J Obstet Gynecol 198(528):e521-e525

6. Ferrara A, Peng T, Kim C (2009) Trends in postpartum diabetes screening and subsequent diabetes and impaired fasting glucose among women with histories of gestational diabetes mellitus: a report from the Translating Research Into Action for Diabetes (TRIAD) Study. Diabetes Care 32:269-274

7. Baker AM, Brody SC, Salisbury K, Schectman R, Hartmann KE (2009) Postpartum glucose tolerance screening in women with gestational diabetes in the state of North Carolina. N C Med J 70:14-19
8. Knowler WC, Barrett-Connor E, Fowler SE et al (2002) Reduction in the incidence of type 2 diabetes with lifestyle intervention or metformin. N Engl J Med 346:393-403

9. Ratner RE, Christophi CA, Metzger BE et al (2008) Prevention of diabetes in women with a history of gestational diabetes: effects of metformin and lifestyle interventions. J Clin Endocrinol Metab 93: 4774-4779

10. McGovern A, Butler L, Jones S et al (2014) Diabetes screening after gestational diabetes in England: a quantitative retrospective cohort study. Br J Gen Pract 64:e17-e23

11. Edwards AG, Naik G, Ahmed H, et al (2013) Personalised risk communication for informed decision making about taking screening tests. Cochrane Database Syst Rev (2):CD001865. doi:10.1002/ 14651858.CD001865.pub3

12. Kengne AP, Beulens JW, Peelen LM et al (2014) Non-invasive risk scores for prediction of type 2 diabetes (EPIC-InterAct): a validation of existing models. Lancet Diabetes Endocrinol 2:19-29

13. Kwak SH, Choi SH, Jung HS et al (2013) Clinical and genetic risk factors for type 2 diabetes at early or late post partum after gestational diabetes mellitus. J Clin Endocrinol Metab 98:E744-E752

14. Gobl CS, Bozkurt L, Prikoszovich T, Winzer C, Pacini G, KautzkyWiller A (2011) Early possible risk factors for overt diabetes after gestational diabetes mellitus. Obstet Gynecol 118:71-78

15. Wang Y, Chen L, Horswell R et al (2012) Racial differences in the association between gestational diabetes mellitus and risk of type 2 diabetes. J Womens Health (Larchmt) 21:628-633

16. Golden SH, Bennett WL, Baptist-Roberts K et al (2009) Antepartum glucose tolerance test results as predictors of type 2 diabetes mellitus in women with a history of gestational diabetes mellitus: a systematic review. Gend Med 6(Suppl 1):109-122

17. Gunderson EP, Hedderson MM, Chiang V et al (2012) Lactation intensity and postpartum maternal glucose tolerance and insulin resistance in women with recent GDM: the SWIFT cohort. Diabetes Care 35:50-56

18. Baptiste-Roberts K, Barone BB, Gary TL et al (2009) Risk factors for type 2 diabetes among women with gestational diabetes: a systematic review. Am J Med 122:207-214.e204

19. Cheung NW, Helmink D (2006) Gestational diabetes: the significance of persistent fasting hyperglycemia for the subsequent development of diabetes mellitus. J Diabetes Complicat 20:21-25

20. Oldfield MD, Donley P, Walwyn L, Scudamore I, Gregory R (2007) Long term prognosis of women with gestational diabetes in a multiethnic population. Postgrad Med J 83:426-430

21. Tura A, Grassi A, Winhofer Y et al (2012) Progression to type 2 diabetes in women with former gestational diabetes: time trajectories of metabolic parameters. PLoS One 7:e50419

22. Moher D, Liberati A, Tetzlaff J, Altman DG (2009) Preferred reporting items for systematic reviews and meta-analyses: the PRISMA statement. BMJ (Clin Res Ed) 339:b2535

23. Wells G, Shea B, O'Connell D et al The Newcastle-Ottawa Scale (NOS) for assessing the quality of nonrandomized studies in metaanalysis. Available from www.ohri.ca/programs/clinical epidemiology/oxford.asp, accessed 20 Sept 2014

24. Wilson A, Lissauer D, Thangaratinam S, Khan KS, MacArthur C, Coomarasamy A (2011) A comparison of clinical officers with medical doctors on outcomes of caesarean section in the developing world: meta-analysis of controlled studies. BMJ (Clin Res Ed) 342: d2600

25. Chinn S (2000) A simple method for converting an odds ratio to effect size for use in meta-analysis. Stat Med 19:3127-3131

26. Cochrane (2012) Review Manager (RevMan) version 5.2. The Nordic Cochrane Centre, Copenhagen

27. Lin PC, Hung CH, Huang RD, Chan TF (2015) Predictors of type 2 diabetes among Taiwanese women with prior gestational diabetes mellitus. Jpn J Nurs Sci 13:3-9 
28. Eades CE, Styles M, Leese GP, Cheyne H, Evans JM (2015) Progression from gestational diabetes to type 2 diabetes in one region of Scotland: an observational follow-up study. BMC Pregnancy Childbirth 15:11

29. Bao W, Yeung E, Tobias DK et al (2015) Long-term risk of type 2 diabetes mellitus in relation to BMI and weight change among women with a history of gestational diabetes mellitus: a prospective cohort study. Diabetologia 58:1212-1219

30. Carvalho Ribeiro AM, Nogueira-Silva C, Melo-Rocha G, Pereira ML, Rocha A (2015) Gestational diabetes: determination of risk factors to diabetes mellitus. Rev Port Endocrinol Diabetes Metab 10:8-13 [article in Portuguese]

31. Feig DS, Shah BR, Lipscombe LL et al (2013) Preeclampsia as a risk factor for diabetes: a population-based cohort study. PLoS Med 10:e1001425

32. Chew WF, Rokiah P, Chan SP, Chee WSS, Lee LF, Chan YM (2012) Prevalence of glucose intolerance, and associated antenatal and historical risk factors among Malaysian women with a history of gestational diabetes mellitus. Singap Med J 53:814-820

33. Mukerji G, Chiu M, Shah BR (2012) Impact of gestational diabetes on the risk of diabetes following pregnancy among Chinese and South Asian women. Diabetologia 55:2148-2153

34. Xiang AH, Li BH, Black MH et al (2011) Racial and ethnic disparities in diabetes risk after gestational diabetes mellitus. Diabetologia 54:3016-3021

35. Ekelund M, Shaat N, Almgren P, Groop L, Berntorp K (2010) Prediction of postpartum diabetes in women with gestational diabetes mellitus. Diabetologia 53:452-457

36. Ogonowski J, Miazgowski T (2009) The prevalence of 6 weeks postpartum abnormal glucose tolerance in Caucasian women with gestational diabetes. Diabetes Res Clin Pract 84:239-244

37. Hossein-nezhad A, Mirzaei K, Maghbooli Z, Larijani B (2009) Maternal glycemic status in GDM patients after delivery. Iran J Diabetes Lipid Disord 8:95-104

38. Russell C, Dodds L, Armson BA, Kephart G, Joseph KS (2008) Diabetes mellitus following gestational diabetes: role of subsequent pregnancy. BJOG 115:253-259

39. Rivero K, Portal VL, Vieira M, Behle I (2008) Prevalence of the impaired glucose metabolism and its association with risk factors for coronary artery disease in women with gestational diabetes. Diabetes Res Clin Pract 79:433-437

40. Krishnaveni GV, Hill JC, Veena SR et al (2007) Gestational diabetes and the incidence of diabetes in the 5 years following the index pregnancy in South Indian women. Diabetes Res Clin Pract 78:398-404

41. Zonenberg A, Telejko B, Topolska J et al (2006) Factors predisposing to disturbed carbohydrate tolerance in patients with previous gestational diabetes mellitus. Diabetol Dosw Klin 6:143-150

42. Weijers RNM, Bekedam DJ, Goldschmidt HMJ, Smulders YM (2006) The clinical usefulness of glucose tolerance testing in gestational diabetes to predict early postpartum diabetes mellitus. Clin Chem Lab Med 44:99-104

43. Kousta E, Efstathiadou Z, Lawrence NJ et al (2006) The impact of ethnicity on glucose regulation and the metabolic syndrome following gestational diabetes. Diabetologia 49:36-40

44. Kim SH, Kim MY, Yang JH et al (2011) Nutritional risk factors of early development of postpartum prediabetes and diabetes in women with gestational diabetes mellitus. Nutrition 27:782-788

45. Jarvela IY, Juutinen J, Koskela P et al (2006) Gestational diabetes identifies women at risk for permanent type 1 and type 2 diabetes in fertile age: predictive role of autoantibodies. Diabetes Care 29:607-612

46. Hunger-Dathe W, Mosebach N, Samann A, Wolf G, Muller UA (2006) Prevalence of impaired glucose tolerance 6 years after gestational diabetes. Exp Clin Endocrinol Diabetes 114:11-17

47. Cho NH, Jang HC, Park HK, Cho YW (2006) Waist circumference is the key risk factor for diabetes in Korean women with history of gestational diabetes. Diabetes Res Clin Pract 71:177-183
48. Lin CH, Wen SF, Wu YH, Huang YY, Huang MJ (2005) The postpartum metabolic outcome of women with previous gestational diabetes mellitus. Chang Gung Med J 28:794-800

49. Schaefer-Graf UM, Buchanan TA, Xiang AH, Peters RK, Kjos SL (2002) Clinical predictors for a high risk for the development of diabetes mellitus in the early puerperium in women with recent gestational diabetes mellitus. Am J Obstet Gynecol $186: 751-756$

50. Dalfra MG, Lapolla A, Masin M et al (2001) Antepartum and early postpartum predictors of type 2 diabetes development in women with gestational diabetes mellitus. Diabetes Metab 27:675-680

51. Pallardo F, Herranz L, Garcia-Ingelmo T et al (1999) Early postpartum metabolic assessment in women with prior gestational diabetes. Diabetes Care 22:1053-1058

52. Buchanan TA, Xiang AH, Kjos SL, Trigo E, Lee WP, Peters RK (1999) Antepartum predictors of the development of type 2 diabetes in Latino women 11-26 months after pregnancies complicated by gestational diabetes. Diabetes 48:2430-2436

53. Steinhart JR, Sugarman JR, Connell FA (1997) Gestational diabetes is a herald of NIDDM in Navajo women. High rate of abnormal glucose tolerance after GDM. Diabetes Care 20:943-947

54. Greenberg LR, Moore TR, Murphy H (1995) Gestational diabetes mellitus: antenatal variables as predictors of postpartum glucose intolerance. Obstet Gynecol 86:97-101

55. Damm P, Kuhl C, Bertelsen A, Molsted-Pedersen L (1992) Predictive factors for the development of diabetes in women with previous gestational diabetes mellitus. Am J Obstet Gynecol 167:607-616

56. Liu H, Zhang C, Zhang S et al (2014) Prepregnancy body mass index and weight change on postpartum diabetes risk among gestational diabetes women. Obesity 22:1560-1567

57. Capula C, Chiefari E, Vero A, Foti DP, Brunetti A, Vero R (2014) Prevalence and predictors of postpartum glucose intolerance in Italian women with gestational diabetes mellitus. Diabetes Res Clin Pract 105:223-230

58. Bentley-Lewis R, Dawson DL, Wenger JB, Thadhani RI, Roberts DJ (2014) Placental histomorphometry in gestational diabetes mellitus: the relationship between subsequent type 2 diabetes mellitus and race/ethnicity. Am J Clin Pathol 141: 587-592

59. Kim C, Newton KM, Knopp RH (2002) Gestational diabetes and the incidence of type 2 diabetes: a systematic review. Diabetes Care $25: 1862-1868$

60. Garber AJ (2012) Obesity and type 2 diabetes: which patients are at risk? Diabetes Obes Metab14:399-408

61. Bakker LE, Sleddering MA, Schoones JW, Meinders AE, Jazet IM (2013) Pathogenesis of type 2 diabetes in South Asians. Eur J Endocrinol 169:R99-R114

62. Meigs JB, Cupples LA, Wilson PW (2000) Parental transmission of type 2 diabetes: the Framingham Offspring Study. Diabetes 49: 2201-2207

63. Chan JC, Malik V, Jia W et al (2009) Diabetes in Asia: epidemiology, risk factors, and pathophysiology. JAMA 301:2129-2140

64. Much D, Beyerlein A, Rossbauer M, Hummel S, Ziegler AG (2014) Beneficial effects of breastfeeding in women with gestational diabetes mellitus. Mol Metab 3:284-292

65. WHO (2013) Diagnostic Criteria and Classification of Hyperglycaemia First Detected in Pregnancy. World Health Organization, Geneva

66. Pierce M, Modder J, Mortagy I, Springett A, Hughes H, Baldeweg S (2011) Missed opportunities for diabetes prevention: postpregnancy follow-up of women with gestational diabetes mellitus in England. Br J Gen Pract 61:e611-e619

67. Middleton P, Crowther CA (2014) Reminder systems for women with previous gestational diabetes mellitus to increase uptake of testing for type 2 diabetes or impaired glucose tolerance. Cochrane Database Syst Rev (3):CD009578. doi: 10.1002/14651858.CD009578.pub2 
68. Kim C, McEwen LN, Piette JD, Goewey J, Ferrara A, Walker EA (2007) Risk perception for diabetes among women with histories of gestational diabetes mellitus. Diabetes Care 30:2281-2286
69. Lambert PC, Sutton AJ, Abrams KR, Jones DR (2002) A comparison of summary patient-level covariates in meta-regression with individual patient data meta-analysis. J Clin Epidemiol 55:86-94 